University of Massachusetts Amherst

ScholarWorks@UMass Amherst

Chemistry Department Faculty Publication Series

Chemistry

1987

\title{
Analytical Information from Doublet Peaks in Flow Injection Analysis Part II. Determination of Stability Constants
}

Julian Tyson

University of Massachusetts Amherst

Follow this and additional works at: https://scholarworks.umass.edu/chem_faculty_pubs

Part of the Chemistry Commons

\section{Recommended Citation}

Tyson, Julian, "Analytical Information from Doublet Peaks in Flow Injection Analysis Part II. Determination of Stability Constants" (1987). Analyst. 1381.

Retrieved from https://scholarworks.umass.edu/chem_faculty_pubs/1381 


\title{
Analytical Information from Doublet Peaks in Flow Injection Analysis Part II.* Determination of Stability Constantst
}

\author{
Julian F. Tyson \\ Department of Chemistry, University of Technology, Loughborough, Leicestershire LE11 3TU, UK
}

\begin{abstract}
An equation was derived for the determination of a stability constant from a flow injection titration experiment in which the product of the reaction is monitored. This equation showed that if the product concentration at the equivalence points of the titration was used as the basis for the calculation, the method was independent of all the variables associated with the flow injection system, provided that a single-line manifold was used. If the system corresponded to that described by the well stirred mixing chamber model, then the time interval between the peaks could be used to determine the ratio of the stoicheiometric coefficients of the reactants. The use of the method is illustrated for the copper(II) - EDTA system giving values for $\log K$ in reasonable agreement with the literature values.
\end{abstract}

Keywords: Flow injection analysis; doublet peak; stability constant; copper(II); EDTA

The determination of stability constants by solution spectrophotometric methods is well established.1,2 Many of these are based on experiments in which the concentrations of reactants are varied in a systematic manner and the extent of the reaction is measured. Viewed in this way, the basic flow injection experiment is a continuous production of the information normally produced by interpolation between the discrete data points of the method of continuous variation (or Job's method). If a single-line manifold is used, all that is required is that a continuous monitor of the dispersion coefficient, $D_{\mathrm{g}}$, is made, as from this, and the known injected sample concentration, $C_{\mathrm{S}}^{\mathrm{s}}$, the concentration of sample before reaction at any point, $C_{\mathrm{g}}^{\mathrm{s}}$, on the dispersed sample profile can be calculated $\left(D_{\mathrm{g}}=C_{\mathrm{o}}^{\mathrm{S}} / C_{\mathrm{g}}^{\mathrm{S}}\right)$. Knowing the concentration of the reagent in the carrier stream, $C_{\mathrm{O}}^{\mathrm{R}}$, allows the corresponding reagent concentration, $C_{\mathrm{g}}^{\mathrm{R}}$, to be calculated as the reagent dispersion coefficient, $D_{\mathrm{g}}^{\mathrm{R}}\left(=C_{\mathrm{O}}^{\mathrm{R}} / C_{\mathrm{g}}^{\mathrm{R}}\right)$, is related to the dispersion by the following equation ${ }^{3}$ :

$$
D_{\mathrm{g}}^{\mathrm{R}}=D_{\mathrm{g}} /\left(D_{\mathrm{g}}-1\right)
$$

Similar equations can be derived for merging-stream manifolds. ${ }^{3}$ These require knowledge of the flow-rate in each stream before concentration profiles can be calculated from the variation of $D_{\mathrm{g}}$ with time. This approach was used recently by Vithanage and Dasgupta ${ }^{4}$ for systems which exhibit isosbestic points. By simultaneously monitoring at an isosbestic wavelength and a reactant and/or product wavelength with a diode array detector, information was obtained on the variation of the dispersion coefficient and the extent of reaction with time.

An alternative approach is to use a gradient-forming device in the manifold that produces known concentration-time profiles. Then the time axis of the data record can be replaced by concentration according to the relationship between them as produced by the particular gradient-forming device. The single well stirred mixing chamber is such a device and its use for determining drug-protein binding constants has been described by Miller and co-workers. 5,6

A relative method, as opposed to the two absolute methods described above, based on the production of a calibration graph for the binding of ligands to a particular metal ion has been described by Yoza et al. ${ }^{7} \mathrm{~A}$ carrier stream of a

\footnotetext{
* For Part I of this series, see page 523.

† Presented at SAC 86, the 7th SAC International Conference on Analytical Chemistry, Bristol, UK, 20-26 July, 1986.
}

pre-formed metal complex with the weakest binding ligand, in the series of compounds of interest, is merged with a water stream into which the next ligand to be investigated is injected. The extent to which the absorbance corresponding to the original metal - ligand complex decreases is a measure of the strength of the binding of the second ligand. By injecting a series of ligands of known binding constant with the metal concerned, a calibration graph is constructed by plotting a function of the peak height against the logarithm of the stability constant. An estimate of the unknown binding constant of a new ligand for that particular metal can be made by interpolations from the calibration graph.

In this paper, the application of the doublet peak flow injection mode to the determination of stability constants is demonstrated for the reaction between copper(II) and EDTA. Doublet peaks are obtained when the operating variables are adjusted so that the injected sample material is in excess over the reagent in the centre of the reaction zone. The basic equations for the time interval between the peaks have been derived ${ }^{3}$ and verified ${ }^{8}$ and the advantages of the technique in terms of extending the conventional working range of a spectrophotometric method discussed. 8

\section{Basis of the Method}

Consider the general reaction

$$
m \mathrm{R}+n \mathrm{~S} \rightleftharpoons \mathrm{P}
$$

where $R, S$ and $P$ are the reagent (in the carrier stream), the sample (i.e., the injected material) and the product which is equivalent to $\mathrm{R}_{m} \mathrm{~S}_{n}$, respectively, and $m$ and $n$ are the stoicheiometric coefficients of $R$ and $S$, respectively. The equilibrium constant for this reaction is given by

$$
K=C_{\mathrm{e}}^{\mathrm{P}} /\left[\left(C_{\mathrm{e}}^{\mathrm{R}}\right)^{m}\left(C_{\mathrm{e}}^{\mathrm{S}}\right)^{n}\right]
$$

where the subscript e denotes the equilibrium concentrations and the activity coefficients are assumed to be unity. At any point along a dispersed sample profile in the flow injection manifold, the concentrations of the sample and the reagent can be obtained from a knowledge of the original concentrations and the dispersion coefficient and hence equation (3) can be rewritten as

$$
K=C_{\mathrm{g}}^{\mathrm{P}} /\left\{\left[\left(C_{\mathrm{o}}^{\mathrm{R}} / D_{\mathrm{g}}^{\mathrm{R}}\right)-m C_{\mathrm{g}}^{\mathrm{P}}\right]^{m}\left[\left(C_{\mathrm{o}}^{\mathrm{S}} / D_{\mathrm{g}}\right)-n C_{\mathrm{g}}^{\mathrm{P}}\right]^{n}\right\}
$$

In the doublet peak mode, there are equivalence points on the rise and fall graphs of the physically dispersed sample and the reagent profiles (see Fig. 1) where the concentrations are in 


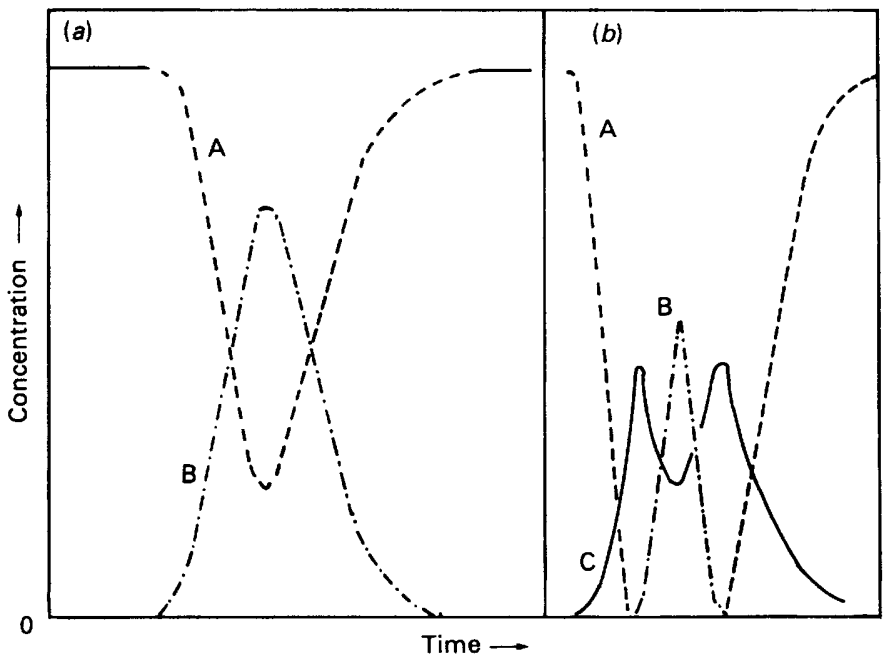

Fig. 1. Formation of the doublet peak. (a) Dispersed reagent (line A) and sample profiles (line $B$ ) in the absence of a chemical reaction. (b) Profiles when a chemical reaction occurs for reagent (line A), sample (line B) and product (line C). The lines are drawn for a 1:1 reaction where the equivalence points in the flow injection titration are located at the intersections of lines $\mathrm{A}$ and $\mathrm{B}$ in $(a)$. The situation obtained with other stoicheiometric coefficients can be readily envisaged

their stoicheiometric equivalence as given by equation (2) and therefore at the particular points represented by the peak maxima of the doublet peak, the following relationship holds:

$$
n C_{\mathrm{eq}_{\mathrm{q}}}^{\mathrm{R}}=m C_{\mathrm{eq}}^{\mathrm{s}}
$$

Hence by substitution of equation (1) in equation (5)

$$
D_{\mathrm{eq}}=1+\left(m C_{\mathrm{o}}^{\mathrm{S}} / n C_{\mathrm{o}}^{\mathrm{R}}\right) \text {. }
$$

and

$$
D_{\mathrm{eq}}^{\mathrm{R}}=1+\left(n C_{\mathrm{o}}^{\mathrm{R}} / m C_{\mathrm{o}}^{\mathrm{S}}\right) \ldots
$$

where the subscript eq refers to the values at the peak maxima of the doublet peaks, i.e., the equivalence points of the FI titration.

Substitution of equations (6) and (7) in equation (4) at the equivalence points gives

$$
\begin{aligned}
K=C_{\mathrm{eq}}^{\mathrm{P}} / & \left(\left\{\left[m C_{\mathrm{o}}^{\mathrm{R}} C_{\mathrm{o}}^{\mathrm{S}} /\left(m C_{\mathrm{o}}^{\mathrm{S}}+n C_{\mathrm{o}}^{\mathrm{R}}\right)\right]\right.\right. \\
& \left.\left.-m C_{\mathrm{eq}}^{\mathrm{P}}\right\}^{m}\left\{\left[n C_{\mathrm{o}}^{\mathrm{R}} C_{\mathrm{o}}^{\mathrm{S}} /\left(m C_{\mathrm{o}}^{\mathrm{S}}+n C_{\mathrm{o}}^{\mathrm{R}}\right)\right]-n C_{\mathrm{eq}}^{\mathrm{P}}\right\}^{n}\right)
\end{aligned}
$$

When $m=n=1$, equation (8) reduces to the simple form

$$
K=C_{\mathrm{eq}}^{\mathrm{P}} /\left(C_{\mathrm{eq}}-C_{\mathrm{eq}}^{\mathrm{P}}\right)^{2}
$$

where $C_{\mathrm{eq}}=C_{\mathrm{o}}^{\mathrm{R}} C_{\mathrm{o}}^{\mathrm{S}} /\left(C_{\mathrm{o}}^{\mathrm{R}}+C_{\mathrm{o}}^{\mathrm{S}}\right)$.

Equation (8) shows that if the concentration of the product is monitored, the value at the top of each doublet peak can be used as the basis for the calculation of $K$, provided that $m$ and $n$ are known. The equation does not depend on what type of gradient-forming device is used, as it applies to any single-line manifold. The ratio of $m / n$ can be determined from the equation for the peak separation. Based on the well stirred mixing tank model, the appropriate equation for a $1: 1$ reaction is ${ }^{8}$

$$
\Delta t_{\text {eq }}=(V / Q) \ln \left[\exp \left(V_{\mathrm{i}} / V\right)-1\right] C_{\mathrm{o}}^{\mathrm{S}} / C_{\mathrm{o}}^{\mathrm{R}} \ldots
$$

For the general $m: n$ reaction, equation (10) is modified to

$$
\Delta t_{\text {eq }}=(V / Q) \ln \left[\exp \left(V_{\mathrm{i}} / V\right)-1\right] m C_{\mathrm{o}}^{\mathrm{S}} / n C_{\mathrm{o}}^{\mathrm{R}}
$$

i.e.,

$\Delta t_{\mathrm{eq}}=(V / Q) \ln \left[\exp \left(V_{\mathrm{i}} / V\right)-1\right] C_{\mathrm{o}}^{\mathrm{S}} / C_{\mathrm{o}}^{\mathrm{R}}+(V / Q) \ln (m / n)$

where $V$ is the volume of the mixing chamber, $Q$ is the volume flow-rate and $V_{\mathrm{i}}$ is the volume injected. A graph of $\Delta t_{\text {eq }}$ versus $(V / Q) \ln \left[\exp \left(V_{\mathrm{i}} / V\right)-1\right] C_{\mathrm{o}}^{\mathrm{S}} / C_{\mathrm{o}}^{\mathrm{R}}$ will have a slope of unity and an intercept of $(V / Q) \ln (m / n)$.
Table 1. Results of determination of stability constant. $C_{\mathrm{o}}^{\mathrm{s}}=8.09 \times$ $10^{-5} \mathrm{M} ; C_{\mathrm{o}}^{\mathrm{R}}=1.41 \times 10^{-4} \mathrm{M} ; C_{\mathrm{eq}}=4.56 \times 10^{-5} \mathrm{M}$

\begin{tabular}{ccccc} 
& $\log$ & & & \\
$\mathrm{pH}$ & $K_{\text {measured }}^{\prime}$ & $\log \alpha_{\mathrm{L}(\mathrm{H})}$ & $\log \alpha_{\mathrm{ML}(\mathrm{H})}$ & $\log K$ \\
3.33 & 6.65 & 10.0 & - & 16.7 \\
2.81 & 5.41 & 11.3 & - & 16.7 \\
2.08 & 5.13 & 13.8 & - & 19.0 \\
1.53 & 5.02 & 15.7 & 1.48 & 19.2 \\
\hline
\end{tabular}

Obviously the procedure can be simplified if a constant flow-rate is assumed over a series of injections, the easiest parameter to vary being $C_{\mathrm{s}}$.

\section{Experimental}

\section{Apparatus}

The flow injection system used was as previously described. ${ }^{8}$ The volume injected was $1410 \mu \mathrm{l}$ and the gradient tube was used as the dispersing device.

\section{Reagents}

Solutions of copper(II) and EDTA of approximately $0.01 \mathrm{M}$ were prepared by dissolving the appropriate amounts of the AnalaR grade salt in distilled water. The solutions were standardised as previously described. 8

\section{Procedure}

At a wavelength $(280 \mathrm{~nm})$ at which the absorbance of the reactants was negligible compared with that of the product, a calibration graph of detector absorbance versus concentration was constructed by serial dilution of a solution containing approximately $1.6 \times 10^{-4} \mathrm{M}$ copper and $10^{-2} \mathrm{M}$ EDTA, buffered with hexammine.

The determination of $K$ was performed at several $\mathrm{pH}$ values. The unbuffered carrier stream was approximately $10^{-4} \mathrm{M}$ EDTA and the injected copper solution approximately $8 \times 10^{-5} \mathrm{M}$. For the first determination, the $\mathrm{pH}$ of the copper solution was adjusted to 3.33 by the addition of small volumes of concentrated hydrochloric acid while monitoring the solution $\mathrm{pH}$ with a glass electrode and $\mathrm{pH}$ meter. Subsequent determinations were made after adjusting the $\mathrm{pH}$ of the injected solution to $2.81,2.08$ and 1.53 . This avoided the search for a non-complexing transparent buffer system which is necessary to avoid over-complicated calculations in what is intended as a demonstration of the feasibility of the technique.

\section{Results and Discussion}

The values of the equilibrium constants calculated from the substitution of the appropriate values into equation (9) are shown in Table 1 . The values are conditional constants that are dependent on the $\mathrm{pH}$. These were corrected by calculation of the side-reaction coefficient values ( $\alpha$ values) at the various $\mathrm{pH}$ values from the data taken from reference 9 , to give the values of $K$ shown in Table 1 . The literature value ${ }^{9}$ for $K$ is $10^{18.8}$ and there is reasonable agreement between this value and the experimentally determined values considering that no attempts were made to buffer the solutions or control ionic strength. The value of the conditional stability constant obtained at $\mathrm{pH} 1.53$ was also corrected for the side reaction of the copper-EDTA complex with protons.

Throughout it has been assumed that the reaction is $1: 1$. The manifold used here would be unsuitable for verification of the ratio of $m / n$, as discussed earlier, as the real value of $V$ does not correspond to the apparent value. However, the data presented in reference 8 can be used to perform such a 
Table 2. Data for determining the ratio $m: n . C_{\mathrm{o}}^{\mathrm{S}}=1.003 \times 10^{-3} \mathrm{M} ; C_{\mathrm{O}}^{\mathrm{R}}=1.012 \times 10^{-4} \mathrm{M}$

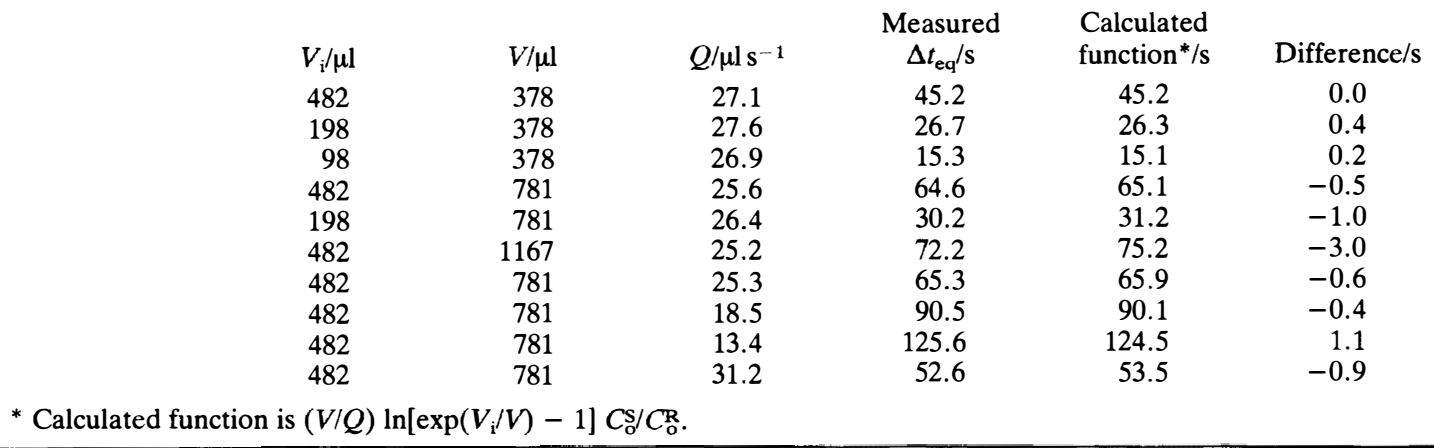

verification. As it was found that small deviations from the well stirred tank behaviour could be attributed to additional dispersion effects in the tail of the injected zone because of the non-negligible effect of the volume of the injection loop, only the data for the injection of $482 \mu$ l or less were used. These values are given in Table 2 ; the mean difference between the measured values of $\Delta t_{\mathrm{eq}}$ and the calculated function [see equation (11)] is -0.39 with a $95 \%$ confidence interval of \pm 0.81 . As this interval includes zero, the data are consistent with the value of $m / n$ being $l$ and hence $\ln (m / n)$ being zero.

This result shows that the method is potentially able to discriminate between a number of simple stoicheiometries, although, as pointed out earlier, the experiment would not be carried out in this fashion.

This method can be contrasted with the slightly more complicated merging-zone method of determining reaction stoicheiometries described by Rios et al. ${ }^{10}$ in which flow injection analogues of the method of continuous variation and the molar-ratio method are devised. The manifold also contains a recirculating loop that produces multiple peaks as the reaction zone passes several times through the detector. The method still requires the conventional graphs to be constructed together with the tangents to the extremes of the graphs. The recirculating system provides data to allow several of these graphs to be constructed for each pair of injections. No attempts were made to calculate the stability constant data from the results obtained.

\section{Conclusion}

The validity of the approach of the doublet peak method for the determination of stability constants has been demonstrated. The method is rapid and, provided that the determination of the reaction stoicheiometry is not required from the same experiment, is, in theory, independent of a number of the experimental variables. The concentration of the product observed at the equivalence points depends only on the concentration of the reagent and the sample and not on the volume injected, the flow-rate, etc. Hence, in principle, the method should yield precise results. The only underlying assumptions are that the system produces dilution of the injected sample solution only with the reagent carrier solution, and that the reaction is rapid.

Financial support from the SERC for the purchase of equipment is gratefully acknowledged.

\section{References}

1. Rossotti, F. J. C., and Rossotti, H., "The Determination of Stability Constants," McGraw-Hill, New York, 1961

2. Albert, A., and Serjeant, E. P., "The Determination of Ionization Constants," Third Edition, Chapman and Hall, London, 1984.

3. Tyson, J. F., Anal. Chim. Acta, 1986, 179, 131.

4. Vithanage, R. S., and Dasgupta, P. K., Anal. Chem., 1986, 58, 326.

5. Abdullahi, G. L., and Miller, J. N., Analyst, 1985, 110, 1271

6. Miller, J. N., Abdullahi, G. L., Sturley, H. N., Gossain, V., and McCluskey, P. L., Anal. Chim. Acta, 1986, 179, 81.

7. Yoza, N., Miyaji, T., Hirai, Y., and Ohashi, S., J. Chromatogr., 1984, 283, 89.

8. Tyson, J. F., Analyst, 1987, 112, 523.

9. Inczedy, J., "Analytical Applications of Complex Equilibria," Ellis Horwood, Chichester, 1976.

10. Ríos, A., Luque de Castro, M. D., and Valcárcel, M., J. Chem. Educ., 1986, 63, 552.

NorE-Reference 8 is to Part I of this series.

Paper A6/358

Received September 22nd, 1986 Accepted November 19th, 1986 